\title{
Prévision de l'énergie du lait de brebis à partir de différents résultats d'analyses : proposition de lait standard pour les brebis laitières
}

\author{
F Bocquier 1, F Barillet 2, P Guillouet 2, M Jacquin 2 \\ 1 INRA, laboratoire sous-nutrition des ruminants, 63122 Saint-Genès-Champanelle; \\ 2 INRA Saga, BP 27, 31326 Castanet-Tolosan Cedex, France
}

(Reçu le 6 janvier 1992; accepté le 5 juin 1992)

\begin{abstract}
Résumé - L'objectif de ce travail était de fournir des équations de prévision de l'énergie du lait de brebis à partir des résultats d'analyses et de proposer également une procédure de standardisation du lait de brebis. Des échantillons (365) de lait de brebis Lacaune ont été prélevés à plusieurs stades de lactation : 276 provenaient de brebis adultes à 6 stades différents entre 41 et $118 \mathrm{j}$ après la mise bas, et 89 échantillons provenaient de brebis primipares, à 79 et $99 \mathrm{j}$ après la mise bas. Ces échantillons ont été analysés par des méthodes classiques (taux butyreux : Gerber, taux protéique : noir-amido, énergie : calorimétrie) et par spectrophotométrie infrarouge, adaptées au lait de brebis. Les résultats obtenus par infrarouge pour les taux butyreux et protéique ont été comparés à ceux obtenus par les méthodes de référence. Les méthodes infrarouge se sont avérées fiables pour déterminer la composition chimique du lait de brebis. Plusieurs équations de prédiction de la valeur énergétique du lait de brebis sont présentées, selon les résultats analytiques disponibles, ou selon les méthodes utilisées. Les équations disponibles dans la bibliographie pour différentes espèces de ruminants laitiers (brebis et vache) ont été testées avec nos résultats analytiques. En terme de précision, ces équations sont souvent proches des nôtres (1,8 à $3,0 \%$ ). Cependant, des biais importants (max: 19\%) sont observés avec certaines équations établies pour le lait de vache. Avec les équations de prédiction de l'énergie du lait présentées dans cet article, nous proposons une démarche pour calculer la production laitière standard des brebis en utilisant une valeur énergétique standard de 5,0 MN/.
\end{abstract}

énergie du lait / composition du lait / brebis laitières

Summary - Prediction of energetic content of ewe's milk from different chemical analyses: proposal for a standard milk for dairy ewes. The aims of this study were: 1) to establish equations of prediction for ewe's milk energy from chemical analyses; and 2) to propose a procedure for standardization of milk for dairy ewes. Ewe's milk samples (365) were taken at different stages of lactation from Lacaune dairy ewes (276 from adult ewes at 6 stages of lactation, ie from 41 to $118 d$ post-lambing and 89 from primiparous ewes at 79 and 99 d). They were analysed by classical (fat: Gerber; protein: amido-black; energy: calonimetry) and infrared methods (Pro-milk or Multispec) 
adapted to ewe milk. For fat and protein, IR methods were reliable when compared to classical methods; and can be used to measure milk composition for routine determination as well as for research purposes. The prediction of milk gross energy has been made using different equations according to available results (fat, protein, lactose) and to the analytical method (classical or IR). Previously published equations for different species (ewe, cow and multi-species) were tested with our data. The range of precision was often close to ours (1.8-3.0\%), but there were important biases with some cow's milk equations ( $\max =19 \%$ ). Based on these equations (table III) of prediction of milk energy content, we propose a procedure for the calculation of a standard milk for dairy-ewes (eSM), on the basis of a standard caloric value of $5.0 \mathrm{MJ} /$.

milk gross energy / milk composition / dairy ewe

\section{INTRODUCTION}

La composition chimique du lait de brebis est soumise à de nombreux facteurs de variations liés à l'animal (race, stade et rang de lactation) mais également au milieu (alimentation, conduite d'élevage, santé, photopériode et température ambiante). Ainsi, par exemple, le taux butyreux (TB) du lait de brebis varie fortement (de 55 à $90 \mathrm{~g} / \mathrm{l}$ ) entre le début et la fin de lactation (Bocquier et al, 1987). Pour tenir compte, de façon synthétique, des variations de la composition chimique du lait, on peut exprimer la production laitière en lait standardisé, c'est-à-dire ayant le même contenu énergétique $(\mathrm{kJ} / \mathrm{l})$. Cette expression en lait standard est particulièrement intéressante pour calculer les besoins énergétiques de lactation et/ou pour comparer les animaux entre eux. L'objectif de ce travail était donc de prédire le contenu énergétique du lait de brebis à partir de sa composition chimique et de proposer une valeur énergétique de référence pour le calcul du lait standard.

La prévision de l'énergie du lait a été étudiée depuis longtemps (Gaines et Davidson, 1923; Tyrrell et Reid, 1965) et continue de susciter des travaux (Sjaunja et al, 1991) et des discussions (Bickel, 1988). Deux voies d'approche ont été choisies : l'une est basée sur l'utilisation de coefficients caloriques pour chaque consti- tuant organique du lait (Perrin, 1958), l'autre repose sur des relations statistiques entre l'énergie mesurée et la composition chimique du lait. L'approche statistique est généralement adoptée. Chez la brebis, les études ont été essentiellement menées sur du lait de femelles allaitantes et parfois sur des échantillons de taille réduite (ValeraAlvarez et al, 1970; Brett et al, 1972; Geenty et Sykes, 1986). Or la composition chimique du lait de brebis allaitante est fortement dépendante des méthodes de traite (manuelle ou mécanique, avec ou sans injection d'ocytocine; Geenty et Sykes, 1986); ce qui pose des problèmes lors de l'établissement de relations statistiques. Quant aux autres équations de prévision de l'énergie du lait proposées pour différentes espèces animales (Perrin, 1958), elles pourraient également être utilisées, mais elles requièrent des analyses chimiques complètes. De plus, comme le taux butyreux moyen du lait est très différent entre espèces (en moyenne 35,45 et $75 \mathrm{~g} / \mathrm{l}$ respectivement pour les vaches, les chèvres et les brebis) (Treacher, 1985), il ne semblait pas réaliste d'utiliser ces équations sans vérifications préalables.

Le premier objectif de ce travail a consisté à établir des équations de prévision de la valeur énergétique du lait de brebis à partir de résultats d'analyses provenant de différentes méthodes. Le second objectif a été de proposer, comme pour les vaches 
laitières (Gaines et Davidson, 1923), une formule de standardisation qui permette d'exprimer les productions laitières des brebis sur une base comparable.

\section{MATÉRIELS ET MÉTHODES}

Des brebis de race Lacaune, traites mécaniquement, nourries en lots dans des conditions normales d'élevage, ont été choisies selon leur numéro de lactation : 45 brebis multipares et 46 primipares. Les échantillons de lait, respectivement 276 et 89 , ont été prélevés à différents stades de la lactation : $41,62,76,90$, 104 et $118 j$ pour les multipares et 79 et $99 j$ pour les primipares. Ces échantillons prélevés à la traite du matin ont été pour partie congelés à $-20^{\circ} \mathrm{C}$ ou conservés avec du bichromate de potassium. Le mème jour, à la traite du soir, la production laitière a été contrôlée et le lait analysé uniquement par spectrophotométrie dans l'infrarouge.

La composition et l'énergie du lait prélevé à la traite du matin ont été déterminées par des méthodes généralement considérées comme des méthodes de référence (Réf) (taux butyreux: Gerber; ETR = \pm 1 gh, taux protéique : noir-amido; $E T R= \pm 0,3 \mathrm{~g} /$, matière sèche : étuve à $103^{\circ} \mathrm{C}$ pendant $24 \mathrm{~h}$; ETR $= \pm 0,5 \mathrm{~g} /$, cendres : four 550 ${ }^{\circ} \mathrm{C}$ pendant $6 \mathrm{~h}$ et énergie : combustion dans un calorimètre adiabatique IRA-C400 après lyophilisation; $E T R= \pm 0,05 \mathrm{MJ} / \mathrm{l}$ ).

Par ailleurs, tous les échantillons ont été analysés par des méthodes spectrophotométriques dans l'infrarouge. Les taux butyreux et protéique ont été mesurés avec le matériel IR-Pro-Milk/ Foss Electric- Danemark, utilisé en routine dans un laboratoire agréé du contrôle laitier ovin (LIAL-Aurillac). La teneur en lactose a été déterminée séparément avec IR-Multispec (Multispec Inst Wheldrate, England). Les instruments ont été calibrés à l'aide d'échantillons de lait de brebis analysés chimiquement par le laboratoire INRA de Poligny pour les taux butyreux et protéique (Grappin, 1986) et à partir d'échantilions analysés par HPLC pour le lactose. La teneur en urée a été déterminée par une méthode enzymatique/colorimétrique (réactif DMBA). Les résultats ont été analysés par le modèle linéaire généralisé (SAS, 1985).

\section{RÉSULTATS ET DISCUSSION}

\section{Caractéristiques chimiques des échantillons}

La production laitière journalière des brebis de l'échantillon a varié de 0,48 à 3,42 l/j. Pour la traite du matin (tableau I), le taux butyreux (TB) a été compris entre 23,5 et $101,5 \mathrm{~g} / \mathrm{l}$, le taux protéique (TP) entre 42,5 et $75,7 \mathrm{~g} / \mathrm{l}$, le lactose $(T L)$ entre 37,5 et $57,1 \mathrm{~g} / \mathrm{l}$ et l'énergie entre 2890 et $6350 \mathrm{~kJ} / \mathrm{l}$. Ces valeurs sont conformes à celles habituellement rapportées chez les brebis laitières (Casu et al, 1975; Barillet, 1985) ou allaitantes (ARC, 1980; Geenty et Sykes, 1986). Cependant, les amplitudes absolues de ces variations sont importantes, car les mesures ont été faites sur une seule traite (matin); elles sont réduites lorsqu'on considère les variations journalières (deux traites, tableau I).

\section{Relations entre analyses de référence et méthodes infra-rouge}

Les résultats obtenus par les deux méthodes ont été comparés par régression simple. Les pentes des droites sont pratiquement égales à $1,00(T B=1,003 ; T P=$ 0,986 ) et les erreurs résiduelles sont respectivement de 1,2 $\mathrm{g} / \mathrm{l}$ et 1,0 g/l. Cela conduit à une légère perte de précision, de l'ordre de $1 \%$ et $2 \%$, ce qui est en accord avec les résultats obtenus précédemment (Grappin, 1986). Les analyses ayant été faites en plusieurs fois, nous avons testé un effet inter-séries qui n'est pas significatif (ns). Le lactose a été calculé par différence aux autres constituants, il n'a donc pas été comparé aux déterminations infrarouge. 
Tableau I. Caractéristiques des échantillons de lait de brebis Lacaune.

\begin{tabular}{|c|c|c|c|c|}
\hline Variables & Moyenne & Ecart type & Minimum & Maximum \\
\hline Stade de lactation (j) & 83,50 & 24,00 & 36 & 136 \\
\hline Production laitière journalière $(1 / j)$ & 1,41 & 0,56 & 0,48 & 3,42 \\
\hline Matières grasses infrarouge $(g / l)$ & 69,80 & 11,60 & 37,7 & 104,8 \\
\hline Protéines infrarouge $(g / l)$ & 52,80 & 6,30 & 41,4 & 73,9 \\
\hline \multicolumn{5}{|l|}{ Traite du matin } \\
\hline Production laitière (I) & 0,85 & 0,34 & 0,13 & 2,00 \\
\hline Matière sèche $(g /)$ & 179,7 & 16,8 & 133,3 & 232,9 \\
\hline \multicolumn{5}{|l|}{ Matières grasses } \\
\hline Gerber $(g /)$ & 64,9 & 12,9 & 23,5 & 101,5 \\
\hline Infra-rouge $(g /)$ & 63,9 & 12,8 & 23,5 & 102,8 \\
\hline \multicolumn{5}{|l|}{ Protéines } \\
\hline Noir-amido $(g /)$ & 53,2 & 6,2 & 42,5 & 75,7 \\
\hline Infrarouge (g/) & 52,7 & 6,2 & 39,5 & 76,9 \\
\hline Urée $(g /)$ & 0,64 & 0,12 & 0,10 & 0,95 \\
\hline Lactose $(\mathrm{g} /)$ & 47,9 & 3,7 & 37,5 & 57,1 \\
\hline Cendres $(g / l)$ & 9,1 & 0,6 & 7,1 & 11,1 \\
\hline Énergie (kJ//) & 4557 & 573 & 2896 & 6357 \\
\hline
\end{tabular}

\section{Corrélations entre constituants du lait etrou avec l'énergie}

Pour une approche statistique de la prévision de l'énergie du lait, il est souhaitable de disposer d'un échantillon qui permette d'obtenir des relations générales. Un des moyens de s'en assurer est de vérifier que les corrélations entre constituants sont proches de celles obtenues sur des échantillons beaucoup plus importants, comme ceux étudiés en amélioration génétique. Malheureusement, la plupart des équations de prévision de l'énergie du lait, aussi bien pour le lait de vache que de brebis, ont été établies avec des données qui n'étaient pas toujours satisfaisantes si l'on considère ce critère. Gaines (1928) et Tyrrell et Reid (1965) ont obtenu respectivement des corrélations entre les taux de matières grasses et les taux protéiques de $+0,9$ et $+0,27$, alors que Sjaunja et al (1991) ont obtenu une valeur de $+0,76$, voisine de celles généralement observées. Pour les autres constituants, ces derniers auteurs ont également obtenu des corrélations: $R_{(T L X T P)}=-0,48$ et $R_{(T L X T B)}=-0,41$, conformes à celles de la bibliographie (Maijala et Hanna, 1974; Boichard et Bonaïti, 1987).

Pour le lait de brebis, les corrélations publiées sont souvent incomplètes : Valera-Alvarez et al (1970): $R_{(\text {TBXTP) }}=+0,41$ (autres corrélations non rapportées), ou surprenantes : Brett et al (1982) :

$$
R_{(T B x T P)}<0,28, R_{(T L X T P)}=-0,52 \text { et }
$$$$
\left.R_{(T L X T B)}<-0,28\right)
$$

Sur notre échantillon, nous avons observé des corrélations négatives entre le lactose et les autres constituants principaux : $R_{(T L X T B)}=-0,46$ et $R_{(T L X T P)}=-0,50$ et positive entre TB et TP $\left(R_{(T B x T P)}=\right.$ $+0,55)$. Ces relations sont conservées dans les deux sous-échantillons d'observa- 
tions (tableau II) et elles sont en accord avec les corrélations phénotypiques observées chez les brebis (Barillet, 1985; Barillet et Boichard, $\left.1987: R_{(\text {TBxTP })}=+0,53\right)$. La teneur en énergie du lait est hautement corrélée au taux butyreux $(R=+0,97)$ parce que la matière grasse est le constituant le plus énergétique du lait, qu'il est le plus variable et que les taux de matières grasses sont habituellement plus élevés que les taux de protéines $(R=+0,69)$ ou de lactose $(R=-0,43)$. Les corrélations observées dans notre échantillon correspondent à des valeurs générales, cet échantillon semble donc approprié à une exploitation statistique de la prévision de l'énergie du lait (tableau II).

\section{Méthodes de prévision de l'énergie du lait de brebis}

Pour estimer l'énergie du lait à partir d'analyses chimiques, deux approches ont été rapportées dans la littérature. Dans la première, l'énergie est calculée en multipliant la teneur en chacun des constituants par sa valeur calorifique (Perrin, 1958). Dans la seconde, l'énergie est prédite par calcul statistique. Fréquemment adoptée, cette seconde méthode consiste à calculer des équations de régression entre la valeur énergétique mesurée et les analyses chimiques disponibles; c'est celle que nous avons retenue.

Les équations de prévision de l'énergie du lait que nous avons obtenues en utilisant les différents résultats d'analyses (taux butyreux, taux protéique, lactose), sont reportées au tableau III, selon les méthodes employées (Réf ou IR). Le rang des écart types résiduels confirme que les matières grasses (TB, $g / l)$ sont le constituant qui a le plus de poids ( $C V=2,8 \%$ ) et que l'introduction d'autres résultats analytiques améliore légèrement la précision des équations obtenues (TB et TP; CV = $1,9 \%$ ). Les précisions de ces équations sont compatibles avec celles qui ont été publiées précédemment (Valera-Alvarez et al, 1970; Brett et al, 1972; Geenty et Sykes, 1986), cependant, les erreurs résiduelles étaient plus élevées, ie respectivement : $3,0 \%, 2,5 \%$ et $6,0 \%$. Dans un cas

Tableau II. Corrélations phénotypiques * entre les teneurs en constituants du lait de brebis *" : lait des brebis multipares au-dessus de la diagonale $(n=276)$ et ensemble des échantillons sous la diagonale $(n=365)$.

\begin{tabular}{lcccccccc}
\hline & Lait & $\begin{array}{c}\text { Matières } \\
\text { grasses }\end{array}$ & Protéines & Lactose & Urée & Cendres & MS & Énergie \\
& & & & & & & & \\
\hline & 1 & $-0,33$ & $-0,30$ & 0,28 & 0,10 & $-0,20$ & $-0,32$ & $-0,34$ \\
Lait & $-0,34$ & 1 & 0,57 & $-0,43$ & $-0,10$ & 0,28 & 0,94 & 0,96 \\
Matières grasses & $-0,31$ & 0,55 & 1 & $-0,46$ & $-0,01$ & 0,40 & 0,76 & 0,71 \\
Protéines & 0,33 & $-0,46$ & $-0,50$ & 1 & $-0,005$ & $-0,39$ & $-0,35$ & $-0,39$ \\
Lactose & 0,09 & $-0,19$ & $-0,06$ & 0,02 & 1 & 0,005 & $-0,08$ & $-0,07$ \\
Urée & $-0,25$ & 0,26 & 0,37 & $-0,43$ & 0,01 & 1 & 0,31 & 0,28 \\
Cendres & $-0,33$ & 0,95 & 0,75 & $-0,39$ & $-0,16$ & 0,27 & 1 & 0,98 \\
MS & $-0,35$ & 0,97 & 0,69 & $-0,43$ & $-0,17$ & 0,25 & 0,99 & 1 \\
Énergie & & & & & & & & \\
\hline
\end{tabular}

\footnotetext{
* Corrigé des efiets du stade de lactation.
}

** Analyses en référence pour les matières grasses et les protéines ( $g /$ ou kJ//). 
Tableau Ill. Prédiction de l'énergie brute du lait de brebis à partir de la composition chimique obtenue par des analyses de référence $(n=365)$ ou par infrarouge $(n=276)$.

\begin{tabular}{|c|c|c|c|c|c|c|c|}
\hline \multirow[t]{2}{*}{ Équation } & \multirow[t]{2}{*}{ Méthode } & \multicolumn{4}{|c|}{ Coefficient de régression constante } & \multirow[t]{2}{*}{$R^{2}$} & \multirow[t]{2}{*}{ ETR } \\
\hline & & $T B(g / l)$ & $T P(g / l)$ & $T L(g / l)$ & cte & & \\
\hline 1 & Ref & $\begin{array}{c}43,16 \\
( \pm 0,53)\end{array}$ & - & - & $\begin{array}{l}1756,14 \\
( \pm 35,22)\end{array}$ & 0,948 & $\pm 131,32$ \\
\hline 2 & $\mathbf{I R}$ & $\begin{array}{c}43,97 \\
( \pm 0,62)\end{array}$ & - & - & $\begin{array}{l}1721,52 \\
( \pm 41,92)\end{array}$ & 0,947 & $\pm 140,42$ \\
\hline 3 & Ref & $\begin{array}{c}35,54 \\
( \pm 0,57)\end{array}$ & $\begin{array}{c}21,37 \\
( \pm 1,19)\end{array}$ & - & $\begin{array}{l}1112,64 \\
( \pm 44,08)\end{array}$ & 0,972 & $\pm 95,63$ \\
\hline 4 & $\mathbb{R}$ & $\begin{array}{c}36,06 \\
( \pm 0,72)\end{array}$ & $\begin{array}{c}22,02 \\
( \pm 1,53)\end{array}$ & - & $\begin{array}{l}1060,29 \\
( \pm 55,70)\end{array}$ & 0,970 & $\pm 105,98$ \\
\hline 5 & Ref & $\begin{array}{c}37,38 \\
( \pm 0,53)\end{array}$ & $\begin{array}{c}24,23 \\
( \pm 1,07)\end{array}$ & $\begin{array}{c}16,66 \\
( \pm 1,54)\end{array}$ & $\begin{array}{r}41,60 \\
( \pm 106,49)\end{array}$ & 0,979 & $\pm 83,30$ \\
\hline 6 & IR & $\begin{array}{c}37,22 \\
( \pm 0,72)\end{array}$ & $\begin{array}{c}25,83 \\
( \pm 1,56)\end{array}$ & $\begin{array}{c}16,80 \\
( \pm 2,76)\end{array}$ & $\begin{array}{c}-5,29 \\
( \pm 183,41)\end{array}$ & 0,974 & $\pm 99,66$ \\
\hline
\end{tabular}

* Le lactose a èté analysé par infrarouge uniquement.

(Brett et al, 1972), la meilleure équation avait été obtenue en incluant le stade de lactation des brebis (jours). Cela était éventuellement transposable à d'autres brebis allaitantes, mais certainement pas à des brebis laitières. En effet, les durées de lactation sont très différentes : 40 à $60 \mathrm{j}$ pour les brebis allaitantes et 100 à $180 \mathrm{j}$ pour les brebis laitières. En fait, si l'amplitude des variations de TB est analogue pour les 2 types de brebis, les effets du stade de lactation sur la composition du lait ne se manifestent pas au même stade de lactation (Bocquier et al, 1987).

\section{Utilisation d'équations déjà publiées}

L'utilisation d'équations établies pour des brebis allaitantes ou pour d'autres espèces de ruminants avec des compositions chimiques moyennes du lait assez différentes peut conduire à des biais importants. Nous avons comparé l'énergie prédite par ces équations à l'énergie mesurée en utilisant les résultats de nos analyses chimiques. L'énergie ainsi calculée avec les équations obtenues avec du lait de brebis allaitantes est assez proche de nos résultats. Cependant, comme dans certaines études les échantillons étaient de taille réduite, nous voulions le vérifier $(n=285$ : ValeraAlvarez et al, 1970; $n=68$ : Brett et al, 1972; $n=28$ : Geenty et Sykes, 1986). Avec des équations établies pour du lait de vache, en utilisant dans nos données seulement le TB, il y a une surestimation de l'énergie prédite qui est respectivement de $+19 \%$ (Tyrrell et Reid, 1965 ), de $+18 \%$ (Crovetto et Van Der Honing, 1984) et de 
$+1 \%$ (Sjaunja et al, 1991). Que ce soit pour le lait de brebis ou de vache, lorsqu'on utilise simultanément le TB, le TP et le lactose (TL), le biais est toujours réduit : $+2,5 \%$ (Brett et al, 1972) et $-1 \%$ (Sjaunja et al, 1991).

C'est l'équation de prévision qui utilise les coefficients caloriques moyens publiés par Perrin (1958), qui provoque le biais le plus faible $(-0,3 \%)$. Cela n'est pas totalement surprenant, puisque lorsque les équations sont établies avec seulement TB et $T P$, les coefficients de régression partiels ne peuvent pas être considérés comme des valeurs caloriques : ils ne sont que l'ajustement statistique de variables qui sont auto-corrélées (tableau II) et ils peuvent n'avoir aucune signification biologique (voir équations 1 et 2; tableau III). Alors que lorsqu'on utilise les 3 constituants majeurs du lait (TB, TP et TL) ces coefficients sont proches de ceux proposés par Perrin (1958) et discutés par Brett et al (1972) : matières grasses 37,38 $( \pm 0,53)$ vs 37,67 ; protéines $24,23( \pm 1,07)$ vs 23,18 et lactose $16,66( \pm 1,54)$ vs 16,53 $(\mathrm{kJ} / \mathrm{g})$. La même remarque pourrait s'appliquer aux équations récemment proposées par Sjanja et al (1991). Cependant, cette approche calorifique n'est pas applicable lorsqu'on ne dispose que des taux butyreux et protéique. En revanche, elle peut être utilisée, ainsi que nous l'avons fait, comme validation de la méthode statistique.

\section{Standardisation du lait de brebis}

La correction ou la standardisation des productions laitières des vaches ont été fréquemment appliquées dans la bibliographie. Cependant, Bickel (1988) a discuté le fait que la formule de Gaines and Davidson (1923) pour la transformation du lait en fat-corrected milk (FCM) est en fait une correction pour la valeur énergétique (ECM). II a proposé d'utiliser un lait standard (LS) basé sur une valeur énergétique de référence de $3,14 \mathrm{MJ} / \mathrm{kg}$ (soit $750 \mathrm{kcal} / \mathrm{kg}$ ) pour le lait de vache. Le lait corrigé pour les matières grasses (FCM) doit être évité, car même si les matières grasses sont le constituant énergétique majeur du lait, les corrélations avec les autres constituants (TP) sont variables; parfois faibles : 0,27 (Tyrrell et Reid, 1965), d'autres fois anormalement élevées : 0,90 (Gaines et Davidson, 1923), voire opposées avec des rations extrêmes (Nelson et al, 1968).

Si l'on excepte plusieurs propositions de corrections pour la matière grasse (FCM) : 6,0\% (Molina, 1984) et 6,5\% (Pulina et al, 1989), il n'y a pas eu, à notre connaissance, de propositions de lait standard pour les brebis laitières. En accord avec les propositions et remarques de Bickel (1988), le lait standard de brebis ne doit pas être confondu avec le lait corrigé pour la matière grasse, qui peut se calculer directement en rapportant le taux butyreux à un taux de référence, sans utiliser de conversion en énergie.

Pour calculer la production laitière standard des brebis, il faut disposer d'équations de prévision de l'énergie du lait et d'une valeur énergétique de référence pour ce lait de brebis. Pour choisir cette valeur énergétique de référence, nous avons utilisé les résultats de composition chimique du lait, obtenus chez différentes races de brebis laitières, afin que les corrections que nous proposons ne modifient pas fondamentalement les performances moyennes exprimées en production laitière brute. Bien que les conditions d'échantillonnage et les niveaux de production ne soient pas rapportés, nous avons utilisé les valeurs du BIDF (1986, 16 références) qui conduisent à une concentration énergétique 
moyenne de 4,99 MJ/l pour les brebis laitières. En ce qui concerne les brebis allaitantes, la concentration énergétique moyenne calculée à partir des données de l'ARC (1980, 18 références) est de 4,77 $\mathrm{MJ} / \mathrm{l}$. En raison des grandes variations observées au sein de notre échantillon (tableau I), nous proposons de retenir la valeur arrondie de 5,0 MJ/l (soit $1195 \mathrm{kcal} / \mathrm{l}$ ) comme contenu énergétique du lait standard pour les brebis laitières.

\section{Procédure de standardisation du lait de brebis}

Pour un calcul précis de la production laitière standard en énergie (PLSE), il faut d'abord choisir des équations de prévision de l'énergie du lait qui soient fiables. Ce choix dépend des méthodes d'analyses utilisées (référence ou infrarouge, tableau III) et des résultats analytiques disponibles (TB, TP, TL). Les taux butyreux et protéique étant fréquemment disponibles, il est préférable de les utiliser simultanément. Ainsi, l'équation suivante, qui est dérivée de l'équation 3 du tableau III, permet un calcul direct du lait standard.

$$
\begin{aligned}
P L S E(I) & =P L \text { brute }(I) \times(0,0071 \times T B(g / l) \\
& +0,0043 \times T P(g / l)+0,2224)
\end{aligned}
$$

La figure 1 illustre les effets de la standardisation de la production laitière, avec la formule ci-dessus, dans le cas d'une comparaison de deux races de brebis (Lacaune et East-Friesian) conduites dans un même milieu d'élevage (Segessemann, 1992). La production laitière brute totale en $168 \mathrm{j}$ est respectivement de 241 et 234 I (différence + $3 \%$ ), alors qu'en lait standard elle est respectivement de 240 et 198 । (soit $+21 \%$ ). Cela traduit des écarts de composition du lait entre ces 2 races de brebis. L'expression en lait standard permet donc une comparaison directe des quantités d'énergie exportées par la mamelle.

La procédure de standardisation du lait de brebis à $5 \mathrm{MJ} / \mathrm{l}$ permet d'exprimer de manière synthétique les niveaux de production. Elle ne saurait remplacer d'autres expressions de la production en fonction de constituants spécifiques; matières grasses ou matières protéiques du lait.
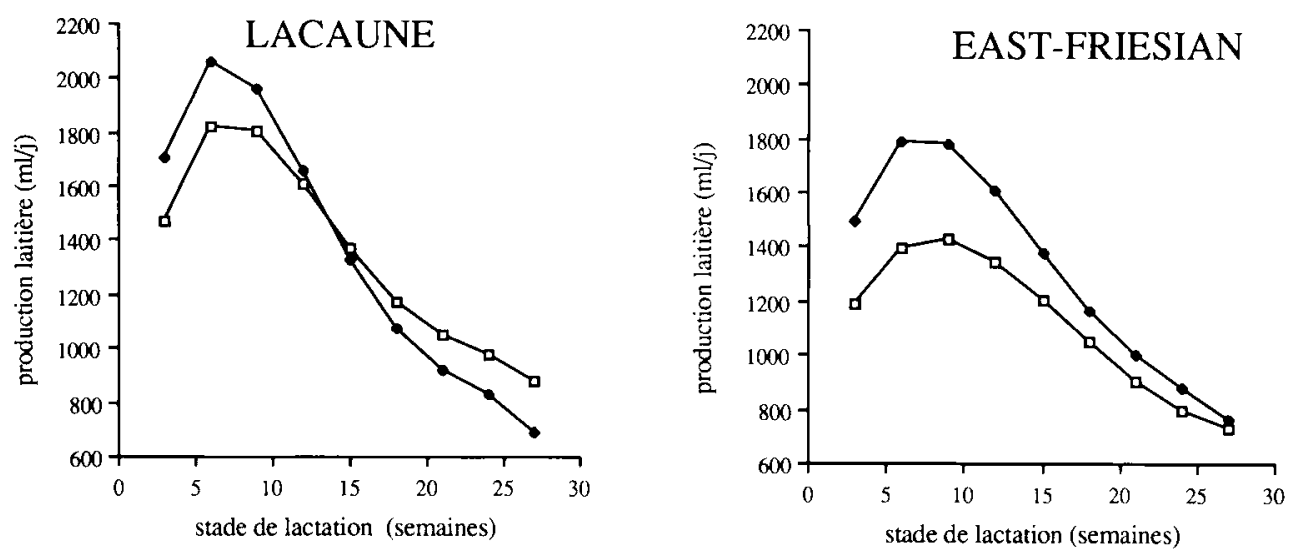

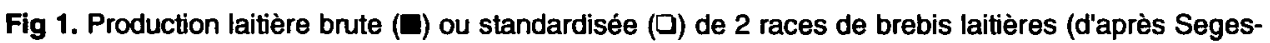
semann, 1992). 


\section{REMERCIEMENTS}

Ce travail a été financé dans le cadre d'une AIP. INRA sur la qualité du lait de brebis. Nous remercions $M$ Retière, directeur du Lial-Aurillac, pour les analyses du lait. Nous remercions également Marie-Rose Aurel pour la collecte des échantillons et $P$ Lebecque pour la détermination de l'énergie du lait.

\section{RÉFÉRENCES}

ARC (1980) Agricultural Research Council: composition of ruminants body and its products. In: The nutrient requirements of ruminant livestock. Commonwealth Agricultural Bureaux, Farnharm Royal, Slough, 43-50

Barillet $F$ (1985) Amélioration génétique de la composition du lait des brebis : l'exemple de la race Lacaune. Thèse de doctorat d'ingénieur, INA Paris-Grignon, 144 p

Barillet F, Boichard D (1987) Studies on dairy productions of milked ewes. I. Estimates of genetic parameters for total milk composition and yield. Genet Sel Evol 19, 459-474

BIDF (1986) Bulletin of the International Dairy Federation, $n^{\circ} 202$, Brussels. Proceedings of the IDF seminar on production and utilization of ewes' and goats' milk. Athens, Greece, 2325 Sept 1985

Bickel H (1988) Fat-corrected milk (FCM) an unaccurate and confusing term. Livest Prod Sci 18, 311-313

Bocquier $F$, Thériez $M$, Brelurut A (1987) Recommandations alimentaires pour les brebis en lactation. Bull Tech CRZV-Theix INRA 70, 199-211

Boichard D, Bonaïti B (1987) Genetic parameters for first lactation dairy traits in Freisian, Montbéliarde and Normande breeds. Genet Sel Evol 19, 337-350

Brett DJ, Corbett JL, Inskip MW (1972) Estimation of the energy value of ewe milk. Proc Austr Soc Anim Prod 9, 286-291

Casu S, Carta R, Flamant JC (1975) Amélioration génétique de la production laitière des brebis Sardes. Ann Genet Sel Anim 7, 73-90
Crovetto GM, Van Der Honing Y (1984) Prediction of the energy content of milk from Friesian ad Jersey cows with normal and high fat concentration. $J$ Anim Phys Nutr 51, 88-97

Gaines WL (1928) The energy basis of measuring milk yield in dairy cows. III Agric Exp Stn Bull 308

Gaines WL, Davidson FA (1923) Relation between percentage fat content and yield of milk. Univ III Agric Exp Stn Bull 245, 577-621

Geenty KG, Sykes AR (1986) A note on the estimation of milk production in sheep. Anim Prod 43, 171-174

Grappin R (1986) Application of indirect instrumental methods to the measurement of fat and protein content of ewe and goat milk. Proceedings of IDF seminar on production and utilization of ewe and goat milk, Sept 1985, Athens (Bull of IDF, $1986 \mathrm{n}^{\circ}$ 202)

Maijala K, Hanna M (1974) Reliable phenotypic and genetic parameters in dairy cattle. 1st world congress on genetics applied to livestock production, Madrid, 7-11 October 1974, vol 1, 541-563

Molina MP (1984) Influence of lactation type and milking practise on productivity of dairy ewes of Manchega breed (Spanish). Tesina de grado, Facultad Farmacia, Universidad de Valencia, $144 p$

Nelson BD, Ellezey HD, Morgan EB, Allen M (1968) Effects of feeding lactating dairy cows varying forage-to-concentrate ratios. I Dairy Sci 51, 1796-1800

Perrin DR (1958) The calorific value of milk of different species. J Dairy Res 25, 215-220

Pulina G, Serra A, Cannas A, Rossi G (1989) Determinazione e stima del valore energetico di latte di pecore di razza sarda (Ital). SISVI

Segessemann V (1992) Wirtschaftlichkeit des Milkschafhaltung in der Schweizerischen Landwirtschaft. PhD thesis, ETH-Zürich (à paraître)

Sjaunja LO, Baevre L, Junkkarinen L, Pedersen $J$, Setala J (1991) A nordic proposal for an energy corrected milk (ECM) formula. Proceeding of the 27th biennal session of the International Committee for Animal Recording (ICAR). Paris, France, 2-6 July 1990. EAAP Publ 50, 156-157 
Statistical Analysis System Institute, Inc (1985) SAS User's guide. SAS Institute, Inc, Cary NC

Treacher $\Pi$ (1985) Dairy sheep production. in: Milk production in developing countries (AJ Smith, ed). University Press, Edinburgh, 388402
Tyrrell HF, Reid JT (1965) Prediction of energy value of cow's milk. J Dairy Sci 48, 1109-1223

Valera-Alvarez H, Wilson LL, Ruch MC, GarciaGarza E, Simpson MJ (1970) Prediction of the energy value of ewe milk from amount and composition characters. J Dairy Sci 53 , 1783-1786 